\title{
Tolerância ao alumínio em porta-enxertos somaclonais de macieira cultivados em solução nutritiva(1)
}

\author{
Adriana Cibele de Mesquita Dantas ${ }^{(2)}$, Gerson Renan de Luces Fortes ${ }^{(3)}$, João Baptista da Silva ${ }^{(4)}$, \\ Adriano Nunes $\mathrm{Nezi}^{(2)}$ e Alexandre Couto Rodrigues ${ }^{(2)}$
}

Resumo - A macieira (Malus sp.) cultivada em solos ácidos com pH inferior a 5,0 pode apresentar distúrbios nutricionais e inibição de crescimento causados por níveis tóxicos de $\mathrm{Al}$ que podem afetar a absorção do Ca, o qual apresenta estreita correlação com a produção de frutos de melhor qualidade. O objetivo deste trabalho foi verificar a tolerância ao Al em 18 somaclones e três cultivares portaenxertos provenientes da seleção in vitro. As plântulas foram cultivadas durante 45 dias em copos de plástico de $300 \mathrm{~mL}$ contendo solução nutritiva e utilizando-se sílica lavada como substrato. Os valores obtidos foram submetidos à análise multivariada pelos componentes principais e agrupamento hierárquico baseado no método de Ward, o qual revelou ser mais adequado na classificação dos clones quanto à tolerância ao $\mathrm{Al}$ do que os testes de médias. O porcentual de redução na matéria seca da parte aérea $\mathrm{e}$ no número de folhas foram características que melhor possibilitaram a discriminação dos clones. Os clones foram classificados em três grupos: bem sensível ou não tolerante, moderadamente tolerante e tolerante.

Termos para indexação: Malus, variação somaclonal, seleção in vitro, métodos de melhoramento.

\section{Tolerance to aluminium in apple rootstocks somaclones in nutrient solution}

Abstract - The apple trees (Malus sp.) cultivated in soils with $\mathrm{pH}$ below 5.0 may present nutritional disorders and growth restriction due to the presence of high toxic levels of Al that may affect the $\mathrm{Ca}$ absorption, which shows a narrow correlation with fruits of better quality. The aim of this work was to verify the tolerance to $\mathrm{Al}$ in 18 apple rootstocks somaclones and three cultivars originated from in vitro selection. The plantlets were cultivated in $300 \mathrm{~mL}$ plastic glasses with washed silica in nutrient solution for 45 days. The obtained values were submitted to a multivariate analysis by the main components and hierarchical grouping based on Ward method, which showed to be more appropriate to classify the clones with relationship to the tolerance to $\mathrm{Al}$ than the tests of averages. The reduction percentage in the dry matter of the shoots and in the number of leaves were characteristic that best permitted clones discrimination. Three groups of clones could be classified such as: no tolerant, slightly tolerant and tolerant.

Index terms: Malus, somaclonal variation, in vitro selection, breeding methods.

(1) Aceito para publicação em 19 de julho de 2000 Extraído da dissertação de mestrado apresentada pelo primeiro autor à Faculdade de Agronomia Eliseu Maciel (FAEM), Universidade Federal de Pelotas (UFPel).

(2) UFPel, FAEM, Caixa Postal 354, CEP 96001-970 Pelotas, RS. E-mail: acmdantas@bol.com.br, anunesnezi@yahoo.com.br, rcale@ufpel.tche.br

(3) Embrapa-Centro de Pesquisa Agropecuária de Clima Temperado (CPACT), Caixa Postal 403, CEP 96001-970 Pelotas, RS. E-mail: gerson $a$ cpact.embrapa.br

(4) UFPel, FAEM. Bolsista do CNPq.

E-mail: jbsilva@ufpel.tche.br

\section{Introdução}

Os solos das regiões predominantes ao cultivo da macieira em Santa Catarina e, em geral, no Sul do Brasil são ácidos e de baixa fertilidade natural. Esses solos são originados do basalto, ricos em óxidos de ferro e $\mathrm{Al}$ (Freire et al., 1994).

A acidez do solo é um dos fatores limitantes e sua correção é imprescindível na implantação do pomar de macieira. Desta forma, a calagem torna-se indispensável para maximizar a produtividade. Para con- 
tornar essa condição adversa, várias alternativas têm sido sugeridas, como o uso de variedades de portaenxertos tolerantes (Suzuki, 1989).

$\mathrm{O}$ pH do solo considerado melhor para a cultura da macieira situa-se na faixa entre 5,5 a 6,0 (Kotzé \& Du Preez, 1988). No entanto, pH inferior a 5,0, com níveis de Al de 3 a 4 ppm na solução do solo, pode levar a distúrbios nutricionais e restrição ao crescimento ocasionados pela presença de elementos tóxicos como Al e Mn (Kotzé \& Joubert, 1980). Em tais condições, ocorre a toxidez em árvores frutíferas, cujo primeiro efeito é a limitação do crescimento da raiz. As células da epiderme ficam menores, com a parede celular delgada, e as células do córtex ficam menores no eixo longitudinal, afetando o crescimento das árvores e reduzindo a absorção de $\mathrm{Ca}, \mathrm{Mg}, \mathrm{K}, \mathrm{Zn}, \mathrm{Cu}$, $\mathrm{Mn}$ e Fe (Kirkpatrick et al., 1975). Esses fatores inibem a formação normal das raízes, tornando-as engrossadas, inchadas, com coloração marrom, quebradiça e, ocasionalmente, necróticas (Foy, 1992).

A toxidez do Al é um fenômeno complexo. Acredita-se que o Al causa lesões a partir do tempo de exposição, as quais podem estar relacionadas a condições de crescimento, como composição iônica do meio de crescimento, temperatura, espécies de plantas, genótipos, idade, entre outras (Rengel, 1992).

Numerosos estudos têm sido realizados usandose solução nutritiva com o intuito de determinar a tolerância de espécies ou variedades ao Al. A toxidez se manifesta por meio de uma série de sintomas que demonstra o efeito contínuo do Al no crescimento do sistema radicular e da parte aérea, e na absorção e utilização de nutrientes (Fernandes \& Rossiello, 1995). Esses efeitos incluem reduções no peso da matéria seca, no número e no comprimento de raízes e na área radicular, que freqüentemente estão associados a aumentos no raio médio e no volume radicular (Sivaguru et al., 1992). Na parte aérea ocorrem reduções no peso de matéria seca e na altura das plantas (Sivaguru \& Paliwal, 1993).

O objetivo deste trabalho foi estudar o efeito do Al, em solução nutritiva, no crescimento e desenvolvimento da parte aérea e raízes dos somaclones e cultivares porta-enxertos de macieira oriundos da seleção in vitro e classificar os clones quanto à tolerância ao Al.

\section{Material e Métodos}

O trabalho foi realizado na Embrapa-Centro de Pesquisa Agropecuária de Clima Temperado. Foram utilizados 18 somaclones, oriundos da calogênese em meio com Al, e três cultivares de porta-enxertos (M.7, M.9 e M.111) de macieira, submetidos ao enraizamento in vitro, os quais foram mantidos em 30 dias de subcultivo. As brotações com médias aproximadas de $3,0 \mathrm{~cm}$ de altura e $3,0 \mathrm{~cm}$ de raízes foram plantadas em copos de plástico de $300 \mathrm{~mL}$, mantidos em caixas de plástico, contendo cada uma 24 copos. Utilizou-se como substrato sílica lavada. Uma lâmina d'água de solução nutritiva, com aproximadamente $5 \mathrm{~cm}$, foi mantida dentro da caixa, e as folhas foram mantidas umedecidas por meio de pulverização com água destilada mais espalhante. A solução nutritiva utilizada foi a descrita por Smith et al. (1969), com fornecimento de $\mathrm{Al}$ nas concentrações de 0 e $7 \mathrm{mg} \mathrm{L}^{-1}$ de $\mathrm{Al}_{2}\left(\mathrm{SO}_{4}\right)_{3} \cdot 18 \mathrm{H}_{2} \mathrm{O}$ (Braccini et al., 1998). O pH da solução foi mantido a $4,0 \pm 0,2$. As soluções foram armazenadas em condição de temperatura ambiente, em local sombreado e renovadas semanalmente.

No período de aclimatização, as plantas foram mantidas sob lona de plástico (150 micras) e sombrite, com aproximadamente $50 \%$ de sombreamento, em casa de vegetação, já submetidas a solução nutritiva com e sem Al. Após 15 dias, retirou-se inicialmente a lona de plástico, permanecendo somente o sombrite, que foi retirado após uma semana.

O experimento foi realizado no mês de setembro, com avaliações semanais para as variáveis altura de plantas e número de folhas expandidas. Após 45 dias, foi feita a avaliação final das variáveis peso de matéria seca da parte aérea e raízes, número e comprimento de raízes.

Empregou-se o delineamento estatístico inteiramente casualizado, utilizando-se 21 clones ( 18 somaclones e três cultivares de porta-enxertos), e dois níveis de $\mathrm{Al}$, em seis repetições.

Para identificar as características mais importantes na discriminação dos genótipos e agrupá-los quanto à tolerância ao Al, utilizaram-se métodos multivariados. Nessa análise, os dados das características de crescimento foram expressos pelas porcentagens de redução ou aumento causadas pelo $\mathrm{Al}$ em relação à média de uma planta controle, sem Al, permitindo, assim, comparar os efeitos do $\mathrm{Al}$ sobre as diversas características estudadas nos 20 genótipos.

Para a determinação dos componentes principais empregou-se o método de Cruz \& Regazzi (1997), e utilizouse para o agrupamento o método hierárquico de Ward

O estudo da importância relativa das características de crescimento avaliadas por meio das sete variáveis estuda- 
das (altura de plantas, número de folhas expandidas, peso de matéria seca da parte aérea, das raízes e total; e número e comprimento de raízes) foi expresso em porcentagens de redução causadas pelo $\mathrm{Al}$ em relação a uma planta controle, sem Al, ou seja, $\mathrm{RC}(\%)=[$ (crescimento sem Al - crescimento com $\mathrm{Al}$ )/crescimento sem $\mathrm{Al}]$ x 100 . Os valores de $\mathrm{RC}(\%)$ foram submetidos aos métodos de análise multivariada de componentes principais e de agrupamento hierárquico de Ward.

\section{Resultados e Discussão}

Observou-se que a cultivar M.7 e os somaclones M.9 ${ }_{36}$, M.9 $9_{37}$, M.9 ${ }_{38}$, M.9 $9_{39}$, M.7 18 e M.7 17 , em presença de $\mathrm{Al}$, apresentaram maior número de folhas; enquanto o somaclone M.7 19 apresentou o menor (Tabela 1). Entretanto, no porta-enxerto M.7 e em seus somaclones verificou-se a presença de internódios mais curtos, de folhas menores e, em grande número delas, escurecimento necrótico nas margens, apresentando enrolamento. Tais informações assemelham-se às observadas por Pavan \& Bingham
(1982) em relação à deficiência de $\mathrm{P}$, o que condiciona o menor crescimento foliar. De acordo com Marschner (1986), o Al pode afetar diretamente a absorção do $\mathrm{P}$ através de formação de complexo, que se precipitam como fosfato de Al na superfície da raiz, podendo afetar o desenvolvimento da parte aérea.

Um dos principais efeitos do Al na parte aérea foi o encurtamento dos internódios, resultando em altura média menor, como é mostrado na Figura 1. As plantas controles, em geral, tiveram melhor crescimento que aquelas em presença do Al. O mesmo foi observado por Passo \& Ruiz (1995), pesquisando o crescimento da parte aérea do cafeeiro.

Em relação à altura das plantas constatou-se que em presença de Al houve aumento significativo para os somaclones M.7 17 , M.9 39 e M.9 37 e diminuição para M.111, M.111 66 e M.9 (Tabela 1).

Outro sintoma da toxicidade de $\mathrm{Al}$ detectado no sistema radicular foi o aumento do número de raízes laterais (Tabela 1). É provável que o efeito tóxico do Al na destruição das células meristemáticas da raiz principal altere a produção de hormônios que con-

Tabela 1. Número de folhas expandidas, altura de plantas, número e comprimento de raízes de porta-enxertos cultivares e somaclones de macieira submetidos à presença (+Al) e ausência (-Al) de $\mathrm{Al}$ em solução nutritiva. Embrapa-CPACT, Pelotas, RS, 1999(1).

\begin{tabular}{|c|c|c|c|c|c|c|c|c|}
\hline \multirow[t]{2}{*}{ Clone } & \multicolumn{2}{|c|}{ Número de folhas } & \multicolumn{2}{|c|}{ Altura de planta $(\mathrm{cm})$} & \multicolumn{2}{|c|}{ Número de raízes } & \multicolumn{2}{|c|}{ Comprimento de raízes $(\mathrm{cm})$} \\
\hline & $-\mathrm{Al}$ & $+\mathrm{Al}$ & $-\mathrm{Al}$ & $+\mathrm{Al}$ & $-\mathrm{Al}$ & $+\mathrm{Al}$ & $-\mathrm{Al}$ & $+\mathrm{Al}$ \\
\hline M.9 $9_{25}$ & $4,44 \mathrm{cdeA}$ & 4,63ghijA & $1,55 \mathrm{deA}$ & $1,25 \mathrm{efA}$ & $5,99 \mathrm{abcA}$ & 5,44 bcdeA & $7,33 \mathrm{cdeA}$ & $5,25 \mathrm{bcA}$ \\
\hline M. $9_{28}$ & $2,72 \mathrm{gA}$ & $3,67 \mathrm{jA}$ & $1,84 \mathrm{cdA}$ & $1,97 \mathrm{abcA}$ & 4,73abcdA & $6,98 \mathrm{abcdA}$ & 6,16 cdefA & 7,83abcA \\
\hline M.111 44 & $4,09 \mathrm{defg} \mathrm{A}$ & 6,25 bcdefA & $1,29 \mathrm{eA}$ & $1,34 \mathrm{efA}$ & 3,90abcdeB & $8,43 a b c A$ & $6,50 \mathrm{cde} A$ & $8,83 \mathrm{abcA}$ \\
\hline M.111 65 & $3,35 \operatorname{defgA}$ & $4,19 \mathrm{ijA}$ & $1,55 \mathrm{def} A$ & $1,43 \operatorname{def} A$ & $4,46 \mathrm{bcde} A$ & $4,52 \mathrm{bcde} A$ & $8,66 \mathrm{bcde} A$ & $6,50 \mathrm{abcA}$ \\
\hline M.111 66 & 5,55bcdefgA & $2,05 \mathrm{kA}$ & $1,88 \mathrm{cdA}$ & $1,31 \mathrm{fB}$ & $2,98 \mathrm{bcdeA}$ & $1,61 \mathrm{eA}$ & $12,83 \mathrm{abA}$ & $5,50 \mathrm{abcB}$ \\
\hline M.111 & $6,05 \mathrm{abcdefgA}$ & $6,07 \mathrm{bcdefgA}$ & $1,81 \mathrm{cdA}$ & $1,42 \mathrm{efB}$ & $6,65 \mathrm{abA}$ & $9,64 \mathrm{abA}$ & $10,10 \mathrm{abcA}$ & $8,50 \mathrm{abcA}$ \\
\hline M.9. & $4,03 \mathrm{de} A$ & $3,63 \mathrm{jA}$ & $1,65 \mathrm{de} A$ & $1,64 \mathrm{cde} A$ & $2,82 \mathrm{bcde} A$ & $2,92 \mathrm{de} A$ & $7,00 \mathrm{cde} A$ & $5,08 \mathrm{bcA}$ \\
\hline M. $9_{12}$ & 7,31aA & $6,75 \mathrm{bcd} A$ & $2,43 \mathrm{abA}$ & $2,38 \mathrm{aA}$ & $5,80 \mathrm{abcA}$ & $5,37 \mathrm{bcdeA}$ & 9,83 bcdeA & $11,00 \mathrm{aA}$ \\
\hline M. $9_{39}$ & $2,31 \mathrm{gB}$ & $7,06 \mathrm{abcA}$ & $1,19 \mathrm{eB}$ & $2,20 \mathrm{abA}$ & $0,94 \mathrm{efB}$ & $4,89 \mathrm{bcdeA}$ & 3,66defgB & $11,00 \mathrm{aA}$ \\
\hline M. $9_{27}$ & $4,33 \mathrm{cde} A$ & 4,41hijA & $1,55 \mathrm{de} A$ & 1,43 defA & 2,36bcdefA & $3,97 \mathrm{cde} A$ & 7,83bcdeA & 7,91abcA \\
\hline M.7 & 3,93 defA & $8,60 \mathrm{aA}$ & $1,25 \mathrm{eA}$ & 1,60cdefA & $6,75 \mathrm{abB}$ & $12,89 \mathrm{aA}$ & $9,16 \mathrm{abcdA}$ & $3,41 \mathrm{cB}$ \\
\hline M. $7_{19}$ & $6,72 \mathrm{abA}$ & 5,03efghijB & $1,84 \mathrm{cdA}$ & $1,60 \mathrm{cdef} A$ & $8,85 \mathrm{aA}$ & $6,73 \mathrm{bcdA}$ & $9,16 \mathrm{abcdA}$ & $5,08 \mathrm{bcA}$ \\
\hline M. $9_{22}$ & $5,61 \mathrm{bcA}$ & 5,71cdefghA & $2,55 \mathrm{abA}$ & $2,30 \mathrm{abA}$ & 3,71abcdeA & $4,68 \mathrm{bcde} A$ & 7,66bcdeA & $10,16 \mathrm{abA}$ \\
\hline M. $9_{37}$ & $2,80 f g B$ & 4,44hijA & $1,25 \mathrm{eB}$ & $2,13 \mathrm{abA}$ & $2,07 \mathrm{cdefA}$ & $4,50 \mathrm{bcde} A$ & $4,83 \mathrm{cdefg} \mathrm{A}$ & $9,25 \mathrm{abA}$ \\
\hline M.111 46 & $3,95 \operatorname{def} A$ & $3,74 \mathrm{jA}$ & $1,80 \mathrm{cdA}$ & 1,60cdefA & $5,43 \mathrm{abcA}$ & $3,94 \mathrm{cde} A$ & 7,83bcdeA & $6,00 \mathrm{abcA}$ \\
\hline M.9 & $6,82 \mathrm{abA}$ & $6,41 \mathrm{bcde} A$ & $2,72 \mathrm{aA}$ & $1,60 \mathrm{cdefB}$ & $6,77 \mathrm{abA}$ & $3,43 \mathrm{cdeA}$ & $14,33 \mathrm{aA}$ & $9,91 \mathrm{abA}$ \\
\hline M. $9_{56}$ & $5,58 \mathrm{bcA}$ & 4,70ghijA & $2,16 b c A$ & $2,18 \mathrm{abA}$ & $4,15 \mathrm{abcde} A$ & $2,02 \mathrm{eA}$ & $8,50 \mathrm{bcde} A$ & $7,25 \mathrm{abcA}$ \\
\hline M. $9_{38}$ & 3,17 efgB & 5,22defghiA & $1,20 \mathrm{eA}$ & $1,54 \mathrm{cdef} A$ & $1,77 \mathrm{cdef} A$ & $5,06 \mathrm{bcde} A$ & 3,50efgA & $6,33 \mathrm{abcA}$ \\
\hline M. $9_{36}$ & $4,55 \mathrm{cdB}$ & 7,47abA & $1,66 \mathrm{de} A$ & $1,90 \mathrm{bcdA}$ & 4,43abcdeA & 7,56abcdA & 7,50bcdeA & $10,16 \mathrm{abA}$ \\
\hline M. $7_{18}$ & $1,31 \mathrm{hB}$ & $3,72 \mathrm{jA}$ & $1,25 \mathrm{eA}$ & $1,55 \mathrm{cdef} A$ & $1,05 \mathrm{defB}$ & $4,89 \mathrm{bcdeA}$ & $1,16 f g A$ & $3,50 \mathrm{cA}$ \\
\hline M. $7_{17}$ & $1,02 \mathrm{hB}$ & 4,86fghijA & $0,54 \mathrm{fB}$ & $1,62 \mathrm{cdeA}$ & $0,00 \mathrm{fB}$ & $8,23 \mathrm{abcA}$ & $0,00 \mathrm{gB}$ & $6,16 \mathrm{abcA}$ \\
\hline
\end{tabular}

${ }^{(1)}$ Letras iguais não diferem pelo teste Duncan a $5 \%$ de probabilidade; letras minúsculas comparam os clones e as letras maiúsculas são comparações entre a presença e ausência de $\mathrm{Al}$. 

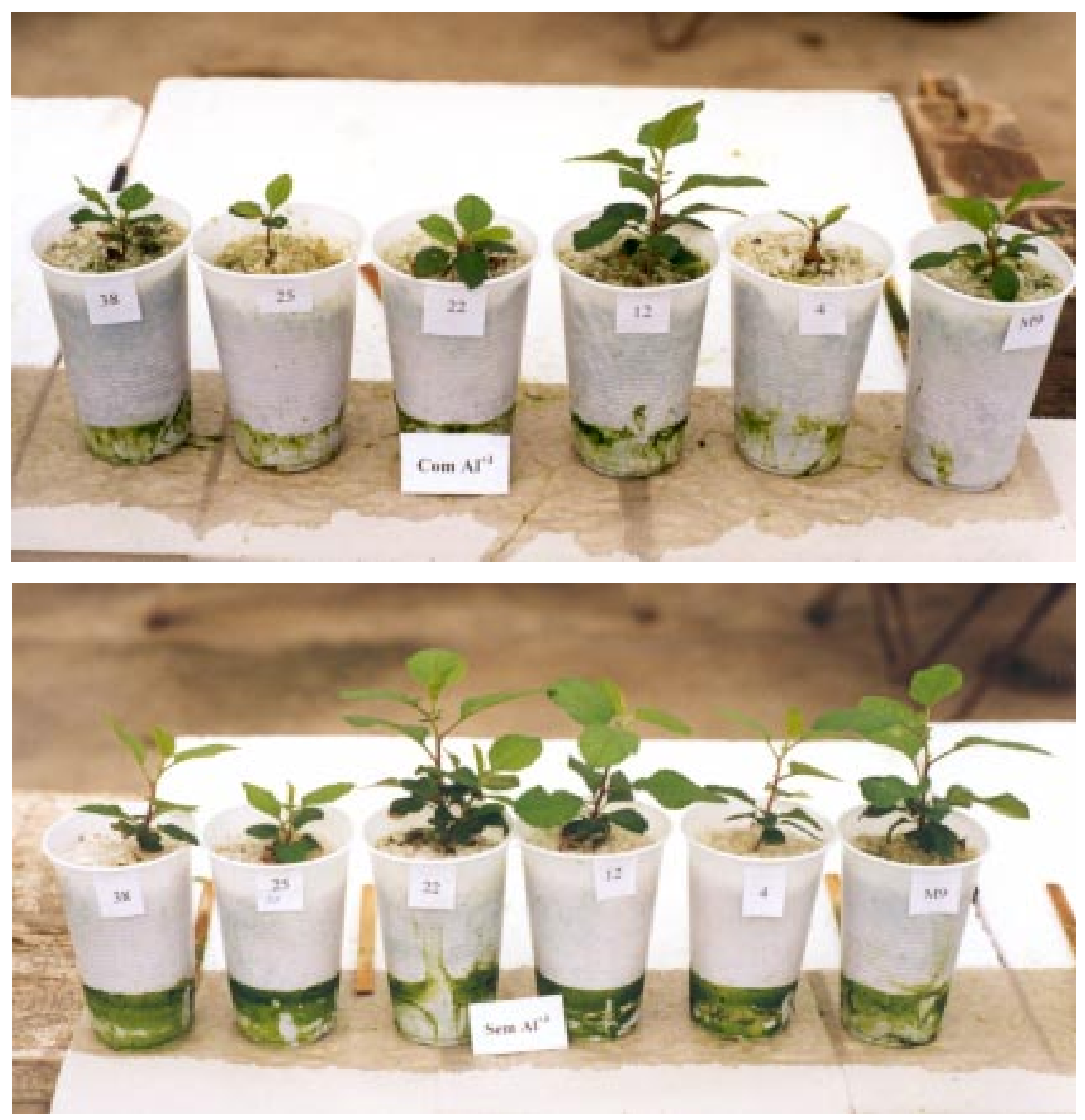

Figura 1. Desenvolvimento da parte aérea do porta-enxerto de macieira cultivar M.9 e cinco somaclones em substrato sem Al e com alumínio. Embrapa-CPACT, Pelotas, RS, 1999.

trolam o crescimento da parte apical (Foy et al., 1972). Segundo Rengel (1992), o Al promove a redução da síntese e do transporte de citocininas nos meristemas da raiz, alterando a direção do movimento acrópeto em vez do basípeto, e aumentando os níveis de ácido abscísico. A perda da dominância apical manifestou-se pela proliferação de raízes laterais nos clones
M.7, M.111 e nos somaclones M.936, M.928, M.7 17 e M. $111_{44}$. Resultados similares foram também observados em genótipos de cafeeiro, por Braccini et al. (1998).

Bennet et al. (1986) relataram, também, que plantas de milho tratadas com Al revelaram alterações morfológicas na raiz, com maior proliferação das raízes 
laterais, sendo este um possível mecanismo para evitar a inibição da absorção de nutrientes.

Considerando que o comprimento das raízes fornece informações seguras da toxidez direta do Al, como encurtamento, ao contrário, verificou-se que os somaclones M.7 17 e M.939 apresentaram maior comprimento relativo na presença desse elemento (Tabela 1).

O Al acumula-se, preferencialmente, no sistema radicular das plantas, translocando-se em pequenas quantidades para a parte aérea (Massot et al., 1992). Esse fato justifica o efeito notável desse íon sobre o sistema radicular, constatado principalmente no porta-enxerto M.9 e em seus somaclones (Figura 2).

Os resultados obtidos neste trabalho mostraram que o comprimento das raízes foi afetado pelo Al. Já Massot et al. (1992) e Baligar et al. (1993) consideram que um dos principais efeitos do Al reside na inibição do crescimento das raízes, que se tornam curtas e grossas. Essa característica, por sinal, serve como o melhor indicador para se avaliar a tolerância ao $\mathrm{Al}$, em solução nutritiva, entre cultivares de diversas espécies.

O Al reduziu significativamente a matéria seca da parte aérea do porta-enxerto M.9 e dos somaclones
M. $9_{39}$ e M. $9_{22}$ (Tabela 2). Resultado semelhante foi observado para a matéria seca total, exceto para o somaclone M.939, que apresentou aumento da matéria seca em presença do Al. Embora a presença do $\mathrm{Al}$ no tecido vegetal cause danos fisiológicos e bioquímicos por vezes irreversíveis e intrinsecamente relacionados ao crescimento de plantas (Pegtel, 1986), os resultados obtidos neste trabalho sugerem que o efeito do $\mathrm{Al}$ sobre a matéria seca pode estar relacionado à própria absorção dos nutrientes. Entretanto, os genótipos testados apresentaram similaridade de comportamento quanto ao teor de matéria seca nas raízes, não diferindo do tratamento com o Al. Observaram-se apenas diferenças de comportamento entre os genótipos. Nascimento et al. (1997) concluíram que as raízes que não apresentaram danos quando expostas ao Al mantiveram a capacidade de assimilação dos nutrientes necessários para a produção de matéria seca.

Observam-se nas decomposições que os clones dos tratamentos com $\mathrm{Al}$, genericamente, foram superiores aos clones dos tratamentos sem Al, demonstrando efeito benéfico do elemento no crescimento das raízes e na parte aérea das plantas. Mesmo fato foi observado por Pereira et al. (1998), em cultivo

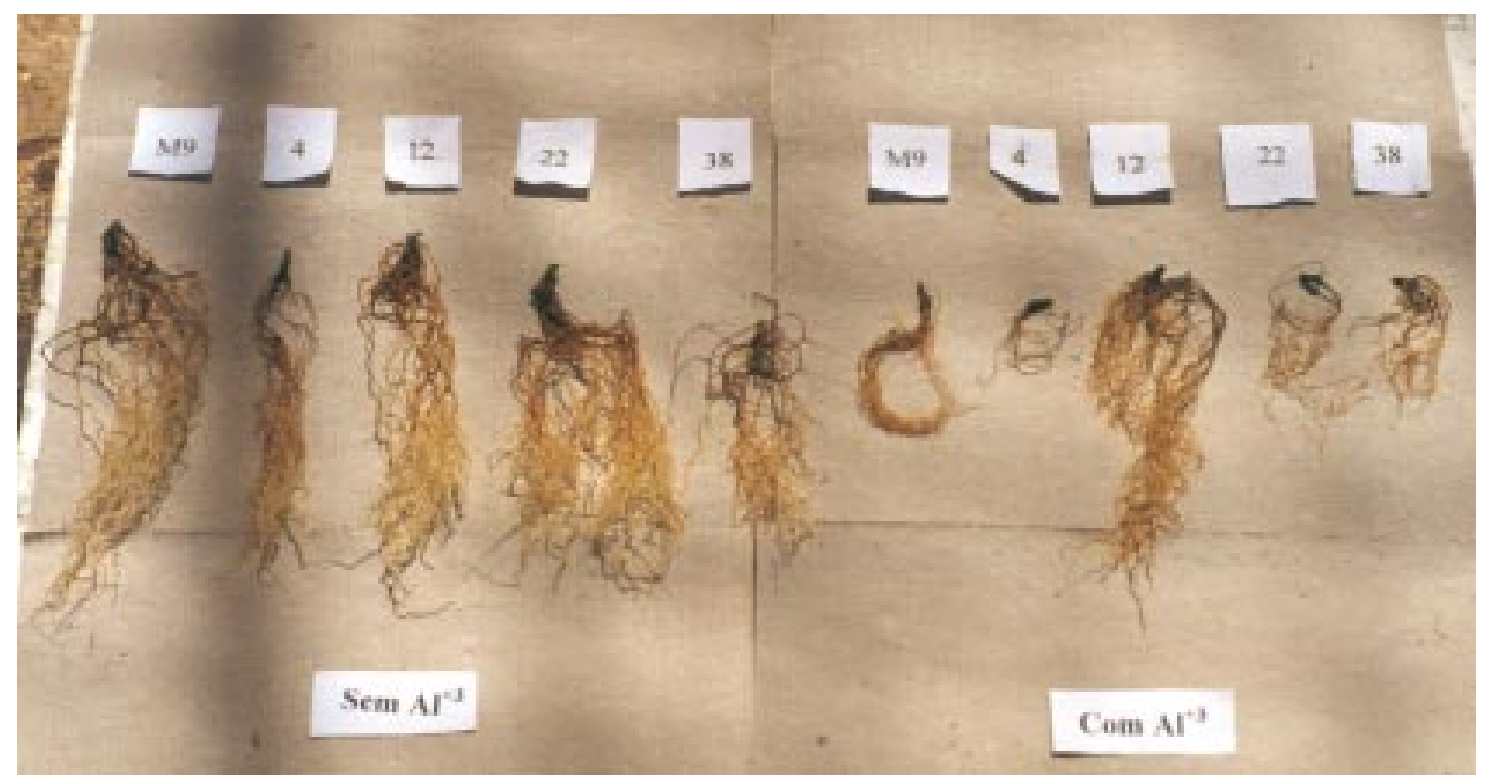

Figura 2. Desenvolvimento do sistema radicular do porta-enxerto de macieira cultivar M.9 e quatro somaclones em substrato sem Al e com alumínio. Embrapa-CPACT, Pelotas, RS, 1999. 
Tabela 2. Matéria seca da parte aérea, raiz e total de porta-enxertos cultivares e somaclones de macieira submetidas à presença (+Al) e ausência (-Al) de Al em solução nutritiva. Embrapa-CPACT, Pelotas, RS, 1999(1).

\begin{tabular}{|c|c|c|c|c|c|c|}
\hline \multirow[t]{2}{*}{ Clone } & \multicolumn{2}{|c|}{ Parte aérea (g/planta) } & \multicolumn{2}{|c|}{ Raiz (g/planta) } & \multicolumn{2}{|c|}{ Total (g/planta) } \\
\hline & $-\mathrm{Al}$ & $+\mathrm{Al}$ & $-\mathrm{Al}$ & $+\mathrm{Al}$ & $-\mathrm{Al}$ & $+\mathrm{Al}$ \\
\hline M. $9_{25}$ & 0,080 cdefA & $0,060 \mathrm{bcdA}$ & $0,020 \mathrm{bcd}$ & $0,022 \mathrm{abc}$ & 0,100 cdefgA & $0,087 \mathrm{bcA}$ \\
\hline M. $9_{28}$ & $0,059 \operatorname{def} A$ & 0,071 abcdA & $0,022 \mathrm{bcd}$ & $0,026 \mathrm{abc}$ & $0,082 \mathrm{defg} \mathrm{A}$ & $0,098 \mathrm{abcA}$ \\
\hline M.111 44 & 0,057 defA & $0,105 \mathrm{abcdA}$ & $0,019 \mathrm{bcd}$ & $0,037 \mathrm{abc}$ & $0,076 \mathrm{defg} \mathrm{A}$ & $0,143 a b c A$ \\
\hline M.111 65 & 0,049 efA & $0,043 \mathrm{cdA}$ & $0,015 \mathrm{~cd}$ & $0,030 \mathrm{abc}$ & $0,065 \mathrm{efgA}$ & $0,073 \mathrm{cA}$ \\
\hline M.11166 & $0,073 \mathrm{cdef} A$ & $0,039 \mathrm{dA}$ & $0,032 \mathrm{abcd}$ & $0,013 \mathrm{c}$ & 0,106 cdefgA & $0,053 \mathrm{cA}$ \\
\hline M.111 & $0,145 \mathrm{bcdA}$ & $0,087 \mathrm{abcdA}$ & $0,036 a b c$ & $0,030 \mathrm{abc}$ & 0,148 bcdefA & $0,117 \mathrm{abcA}$ \\
\hline M. $9_{4}$ & $0,102 \mathrm{cde} A$ & $0,039 \mathrm{dA}$ & $0,023 \mathrm{abcd}$ & $0,043 a b c$ & $0,125 \mathrm{cdef} A$ & $0,053 \mathrm{cA}$ \\
\hline M. $9_{12}$ & $0,198 \mathrm{abA}$ & $0,163 \mathrm{aA}$ & $0,053 \mathrm{a}$ & $0,047 \mathrm{ab}$ & $0,251 \mathrm{abA}$ & $0,208 \mathrm{aA}$ \\
\hline M.9 & $0,043 \mathrm{efA}$ & $0,140 \mathrm{abB}$ & $0,009 \mathrm{~cd}$ & $0,049 \mathrm{a}$ & $0,053 \mathrm{efgB}$ & $0,189 \mathrm{abA}$ \\
\hline M. $9_{27}$ & 0,081 cdefA & $0,058 \mathrm{bcdA}$ & $0,023 \mathrm{abcd}$ & $0,016 \mathrm{bc}$ & 0,104 cdefgA & $0,074 \mathrm{cA}$ \\
\hline M.7 & $0,096 \mathrm{cdefA}$ & $0,119 \mathrm{abcdA}$ & $0,017 \mathrm{~cd}$ & $0,032 \mathrm{abc}$ & 0,113 cdefg $\mathrm{A}$ & $0,153 a b c A$ \\
\hline M. $7_{19}$ & $0,127 b c d e A$ & $0,070 \mathrm{bcdA}$ & $0,037 \mathrm{abc}$ & $0,015 \mathrm{c}$ & 0,165 bcdeA & $0,086 \mathrm{bcA}$ \\
\hline M. $9_{22}$ & $0,164 \mathrm{bcA}$ & $0,078 \mathrm{abcdB}$ & $0,035 \mathrm{abc}$ & $0,023 a b c$ & $0,200 \mathrm{abcA}$ & $0,102 \mathrm{abcB}$ \\
\hline M. $9_{37}$ & 0,087 cdefA & $0,105 \mathrm{abcdA}$ & $0,018 \mathrm{bcd}$ & $0,028 \mathrm{abc}$ & 0,106 cdefgA & $0,134 \mathrm{abcA}$ \\
\hline M.11146 & 0,062 defA & $0,043 \mathrm{cdA}$ & $0,021 \mathrm{bcd}$ & $0,013 \mathrm{c}$ & $0,083 \operatorname{defgA}$ & $0,056 \mathrm{cA}$ \\
\hline M.9 & $0,247 \mathrm{aA}$ & $0,088 \mathrm{abcdB}$ & $0,049 \mathrm{ab}$ & $0,027 \mathrm{abc}$ & $0,297 \mathrm{aA}$ & $0,115 \mathrm{abcB}$ \\
\hline M. $9_{56}$ & $0,157 \mathrm{bcA}$ & $0,135 \mathrm{abcA}$ & $0,038 \mathrm{abc}$ & $0,032 \mathrm{abc}$ & $0,185 \mathrm{bcdA}$ & $0,167 \mathrm{abcA}$ \\
\hline M. $9_{38}$ & 0,053 defA & $0,046 \mathrm{bcdA}$ & $0,012 \mathrm{~cd}$ & $0,019 \mathrm{abc}$ & $0,066 \mathrm{efg} \mathrm{A}$ & $0,066 \mathrm{cA}$ \\
\hline M. $9_{36}$ & 0,078 cdefA & $0,100 \mathrm{abcdA}$ & $0,020 \mathrm{abcd}$ & $0,035 \mathrm{abc}$ & 0,104 cdefgA & $0,135 \mathrm{abcA}$ \\
\hline M. $7_{18}$ & 0,039 efA & $0,005 \mathrm{bcdA}$ & $0,009 \mathrm{~cd}$ & $0,018 \mathrm{abc}$ & $0,049 \mathrm{fgA}$ & $0,074 \mathrm{cA}$ \\
\hline M. $7_{17}$ & $0,004 \mathrm{fA}$ & $0,072 \mathrm{abcdA}$ & $0,001 \mathrm{~d}$ & $0,030 \mathrm{abc}$ & $0,006 \mathrm{gA}$ & $0,098 \mathrm{abcA}$ \\
\hline
\end{tabular}

(1)Letras iguais não diferem pelo teste Duncan a 5\% de probabilidade; letras minúsculas comparam os clones e as letras maiúsculas são comparações entre a presença e ausência de alumínio.

in vitro do porta-enxerto de macieira cultivar Marubakaido, na presença de diferentes concentrações de Al.

Kotzé (1974), estudando seis porta-enxertos de macieira, em solução nutritiva, observou que em cinco deles o crescimento das brotações foi inibido pela presença do Al, e que a cultivar M.111 apresentou nessa condição o seu melhor crescimento com Al.

Segundo Massot et al. (1992), apenas o índice de tolerância baseado na elongação radicular não deve ser um indicador seguro da sensibilidade ao Al. $\mathrm{O}$ crescimento da parte aérea deve ser também considerado, uma vez que danos no sistema radicular podem causar menor crescimento da parte aérea.

Ainda que o Al não seja considerado um elemento essencial, e se desconheçam os mecanismos pelos quais possa, algumas vezes, induzir o crescimento ou produzir outros efeitos benéficos, alguns pesquisadores (Foy, 1974; Marschner, 1986; Kaminski, 1989) discutem a possibilidade de o Al interferir em algumas reações do processo metabólico das plantas. Entretanto, a regulação dos eventos bioquímicos e fisiológicos são determinados geneticamente, e o genótipo das plantas não é caracterizado por um só gene. Nesse particular, há necessidade de maiores estudos em nível genético para se melhor determinar os mecanismos envolvidos na tolerância das diferentes espécies vegetais ao $\mathrm{Al}$.

A análise de componentes principais dos dados permitiu inferir que, com apenas os dois primeiros componentes, foi possível atingir mais de $86 \%$ da variação total (Tabela 3 ). No estudo da importância relativa das características para distinguir os somaclones quanto à tolerância ao $\mathrm{Al}$, foram utilizados os coeficientes de ponderação ou autovetores das características dos componentes principais (Tabela 4). O critério adotado na seleção das características de menor importância consistiu na identificação daquelas que apresentaram os maiores coeficientes de ponderação nos últimos componentes principais. Segundo Cruz \& Regazzi (1997), o descarte das características de maior coeficiente de ponderação deve ser feito a partir do último componente principal até aquele que não exceda ao número índi- 
Tabela 3. Porcentuais de redução ou de aumento do efeito do Al sobre as sete variáveis avaliadas de porta-enxertos cultivares e somaclones de macieira. Embrapa-CPACT, Pelotas, RS, 1999(1).

\begin{tabular}{|c|c|c|c|c|c|c|c|}
\hline Clone & MSPA & MSR & MST & NR & $\mathrm{CR}$ & $\mathrm{NF}$ & AP \\
\hline M.9. & 25,12 & $-7,84$ & 12,88 & 9,18 & $-104,63$ & $-4,27$ & 19,35 \\
\hline M. $9_{28}$ & $-19,89$ & $-16,24$ & $-18,90$ & $-47,56$ & $-27,11$ & $-34,92$ & $-7,06$ \\
\hline M.111 44 & $-84,61$ & $-19,89$ & $-87,58$ & $-116,15$ & $-35,84$ & $-52,81$ & $-3,87$ \\
\hline M.111 65 & 13,25 & $-111,61$ & $-12,25$ & $-1,34$ & 24,94 & $-25,07$ & 8,39 \\
\hline M.111 66 & 46,17 & 57,66 & 50,00 & 45,97 & 57,13 & 63,06 & 39,89 \\
\hline M.111 & 39,95 & 18,47 & 21,15 & $-44,96$ & 15,84 & $-0,33$ & 21,54 \\
\hline M. $9_{4}$ & 61,22 & $-86,01$ & 57,74 & $-3,54$ & 27,42 & 9,92 & 0,60 \\
\hline M.9. & 17,62 & 10,82 & 17,16 & 7,41 & 43,60 & 7,66 & 2,05 \\
\hline M.939 & $-225,01$ & $-400,00$ & $-255,93$ & $-420,20$ & $-200,54$ & $-205,62$ & $-84,87$ \\
\hline M. $9_{27}$ & 28,02 & 29,36 & 28,42 & 68,22 & $-1,02$ & $-1,84$ & 7,74 \\
\hline M7 & $-23,21$ & $-88,23$ & $-34,59$ & $-90,96$ & 62,77 & $-118,82$ & $-28,00$ \\
\hline M.7 19 & 44,93 & 58,99 & 37,65 & 23,95 & 44,54 & $-25,14$ & 13,04 \\
\hline M. $9_{22}$ & 52,43 & 33,51 & 49,07 & $-26,14$ & $-32,63$ & $-1,78$ & 9,80 \\
\hline M.937 & $-21,10$ & $-50,79$ & $-26,48$ & $-117,39$ & $-91,51$ & $-58,87$ & $-70,40$ \\
\hline M. $111_{46}$ & 31,63 & 34,28 & 32,77 & 27,44 & 23,37 & 5,31 & 11,11 \\
\hline M.9 & 64,17 & 44,80 & 61,01 & 49,33 & 30,84 & 6,01 & 41,17 \\
\hline M.9 ${ }_{56}$ & 14,50 & 17,31 & 9,96 & 51,32 & 14,70 & 15,77 & 0,92 \\
\hline M.938 & 12,96 & $-56,69$ & 0,00 & $-185,87$ & $-80,85$ & $-64,66$ & $-28,33$ \\
\hline M.9. & $-26,87$ & $-35,24$ & $-29,80$ & $-70,65$ & $-35,46$ & $-64,17$ & $-14,45$ \\
\hline M. $7_{18}$ & $-42,27$ & $-172,63$ & $-51,83$ & $-365,71$ & $-201,72$ & $-183,96$ & $-24,00$ \\
\hline
\end{tabular}

(1)MSPA: matéria seca da parte aérea; MSR: matéria seca da raiz; MST: matéria seca total; NR: número de raízes; CR: comprimento de raiz; NF: número de folhas; AP: altura da planta.

Tabela 4. Autovalores, variação acumulada relativa dos componentes principais e coeficientes de ponderação das características de crescimento de 20 genótipos de porta-enxertos de macieira, sob efeito do $\mathrm{Al}$ em solução nutritiva. Embrapa-CPACT, Pelotas, RS, 1999.

\begin{tabular}{cccrrrrrrr}
\hline \multirow{2}{*}{$\begin{array}{c}\text { Componentes } \\
\text { principais }\end{array}$} & Autovalor & Variação & \multicolumn{7}{c}{ Características de crescimento $^{(\mathbf{1})}$} \\
\cline { 6 - 10 } & & acumulada & MSPA & \multicolumn{1}{c}{ MSR } & \multicolumn{1}{c}{ MST } & NR & CR & NF & AP \\
\hline CP1 & 5,017610 & 71,68 & $-0,1393$ & 0,4175 & 0,4086 & 0,4251 & 0,3687 & 0,4150 & 0,3879 \\
CP2 & 1,013414 & 86,16 & 0,9350 & $-0,1912$ & $-0,0520$ & 0,1240 & 0,1357 & 0,1912 & 0,1269 \\
CP3 & 0,403961 & 91,93 & $-0,1165$ & $-0,1746$ & $-0,2963$ & 0,1843 & 0,8207 & $-0,1378$ & $-0,3763$ \\
CP4 & 0,253113 & 95,54 & $-0,0893$ & $-0,2237$ & $-0,1463$ & $-0,2591$ & 0,2522 & $-0,3734$ & 0,8065 \\
CP5 & 0,171099 & 97,99 & 0,1430 & $-0,0783$ & 0,8142 & $-0,2757$ & 0,2048 & $-0,3940$ & $-0,1932$ \\
CP6 & 0,088987 & 99,26 & 0,2495 & 0,8377 & $-0,2394$ & $-0,2168$ & 0,0975 & $-0,3467$ & $-0,0441$ \\
CP7 & 0,051815 & 100,00 & $-0,0461$ & 0,0256 & $-0,0289$ & $-0,7615$ & 0,2388 & 0,5978 & $-0,0458$ \\
\hline
\end{tabular}

(1)MSPA: matéria seca da parte aérea; MSR: matéria seca da raiz; MST: matéria seca total; NR: número de raízes; CR: comprimento de raiz; NF: número de folhas; AP: altura da planta.

ce 0,70. Adotando-se este critério, concluiu-se que as características de menor importância foram: número de raízes (NR), matéria seca das raízes (MSR), matéria seca total (MST), altura da planta (AP) e comprimento das raízes (CR). Essas características são dispensáveis ou redundantes por apresentarem alta correlação com as características mais importantes, ou seja, com a matéria seca da parte aérea (MSPA) e com o número de folhas (NF). Diferentemente do que ocorreu com genótipos de cafeeiro, estudados por
Braccini et al. (1998), neste trabalho as características radiculares não se constituíram nas mais sensíveis quanto à toxidez do $\mathrm{Al}$.

Cambraia \& Cambraia (1995) sugerem uma combinação de variáveis, expressas por seus parâmetros biométricos, que podem ser avaliadas pela sua relação direta com o próprio grau de tolerância ao Al.

Pela análise de agrupamento (método hierárquico de Ward) dos dados porcentuais de redução e aumento do efeito do Al sobre as sete variáveis analisadas (Tabela 5), pode-se classificar todo o material 
Tabela 5. Grupos com padrões de comportamentos similares, estabelecidos pelo método de Ward com suas respectivas médias por grupo formado de 20 genótipos de porta-enxertos de macieira. Embrapa-CPACT, Pelotas, RS, 1999.

\begin{tabular}{|c|c|c|c|c|c|c|c|c|}
\hline \multirow[t]{2}{*}{ Grupo } & \multirow[t]{2}{*}{ Genótipo } & \multicolumn{7}{|c|}{ Característica de crescimento $^{(1)}$} \\
\hline & & MSPA & MSR & MST & NR & CR & NF & AP \\
\hline $\mathrm{I}$ & $\begin{array}{l}\text { M.9. } \\
\text { M. M. }{ }_{46}, \text { M.9 } \\
\text { S6 }\end{array}$ & 38,70 & 19,21 & 34,34 & 18,92 & 10,83 & 6,76 & 15,20 \\
\hline II & 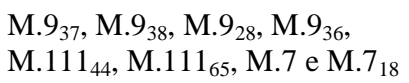 & $-23,96$ & $-68,91$ & $-32,67$ & $-124,45$ & $-40,09$ & $-75,41$ & $-20,96$ \\
\hline III & M.9 $9_{39}$ & $-225,01$ & $-400,00$ & $-255,93$ & $-420,20$ & $-200,54$ & $-205,62$ & $-84,87$ \\
\hline
\end{tabular}

(1)MSPA: matéria seca da parte aérea; MSR: matéria seca da raiz; MST: matéria seca total; NR: número de raízes; CR: comprimento de raiz; NF: número de folhas; AP: altura da planta.

analisado em três grupos. O Grupo I apresentou os maiores porcentuais de redução para MSR, MST, NR, $\mathrm{CR}$, NF e AP, sendo caracterizado como o de maior sensibilidade ao $\mathrm{Al}$ ou não tolerante; foram classificados neste grupo os clones M.9 ${ }_{27}$, M. $111_{46}$, M.956, M.111, M.719, M.94, M.912, M.922, M.9 25, M. $111_{66}$ e M.9. O Grupo II, com menor porcentual de aumento, ficou caracterizado como moderadamente tolerante, e foram incluídos os clones M.9 ${ }_{37}$, M.9.9, M.9 ${ }_{28}$,

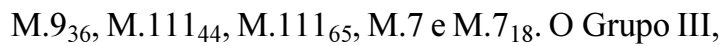
constituído pelo clone M.9 $9_{39}$, mostrou um crescimento maior na presença do Al, destacando-se pelos altos valores em todas as variáveis analisadas, sendo interpretado como tolerante.

\section{Conclusões}

1. A análise multivariada é mais adequada para classificar os clones quanto à tolerância ao $\mathrm{Al}$ do que os testes de médias.

2. Os porcentuais de redução na matéria seca da parte aérea e no número de folhas são as características que melhor possibilitam a discriminação dos clones.

3. A análise de agrupamento, baseada no método de Ward, dá ensejo à separação dos clones nas três classes de tolerância especificadas: não tolerante ou bem sensível, moderadamente tolerante e tolerante.

\section{Agradecimentos}

Aos pesquisadores Cláudio Freire e Maria do Carmo Raseira da Embrapa-Centro de Pesquisa Agropecuária de Clima Temperado, pela contribuição na realização deste trabalho.

\section{Referências}

BALIGAR, V. C.; SCHAFFERT, R. E.; SANTOS, H. L.; PITTA, G. V. E.; BAHIAFILHO, A. F. C. Soil aluminium effects on uptake, influx, and transport of nutrients in sorghum genotypes. Plant and Soil, Dordrecht, v. 150, p. 271-277, 1993.

BENNET, R. J.; BREEN, C. M.; FEY, M. V. Aluminum toxicity and induced nutrient disorders involving the uptake and transport of P, K, Ca and Mg in Zea mays L. South African Journal of Plant and Soil, Pretoria, v. 3, p. 11$17,1986$.

BRACCINI, M. C. L.; MARTINEZ, H. E. P.; PEREIRA, P. R. G.; SAMPAIO, N. F.; SILVA, E. A. M. Tolerância de genótipos de cafeeiro ao $\mathrm{Al}$ em solução nutritiva. I. Crescimento e desenvolvimento da parte aérea e sistema radicular. Revista Brasileira de Ciência do Solo, Campinas, v. 22, p. 435-442, 1998.

CAMBRAIA, J.; CAMBRAIA, M. C. Avaliação de híbridos de milho quanto à tolerância ao alumínio em solução nutritiva. Revista Ceres, Viçosa, v. 4, n. 241, p. 297-307, 1995.

CRUZ, C. D.; REGAZZI, A. J. Modelos biométricos aplicados ao melhoramento genético. Viçosa : UFVImprensa Universitária, 1997. 390 p. 
FERNANDES, M. S.; ROSSIELLO, O. P. R. Mineral nitrogen in plant physiology and plant nutrition. Critical Reviews in Plant Sciences, Boca Raton, v. 14, p. 111148, 1995

FOY, C. D. Effect of aluminum on plant growth. In: CARSON, F. W. (Ed.). The plant root and its environment. Charlottesville : University Press of Virginia, 1974. p. 601-642.

FOY, C. D. Soil chemical factors limiting plant root growth. In: HATFIELD, J. L.; STEWART, B. A. (Ed.). Limitations to plant root growth. New York : Springer, 1992. p. 97-149.

FOY, C. D.; FLEMING, A. L.; GERLOFF, G. C. Differential aluminum tolerance in two snapean varieties. Agronomy Journal, Madison, v. 64, p. 815-818, 1972.

FREIRE, C. J. S.; CAMELATO, D.; CANTILLANO, R. F. F.; KOVALESKI, A.; FORTES, J. F. A cultura da maçã. Brasília : Embrapa-SPI, 1994. 107 p.

KAMINSKI, J. Acidez do solo e a fisiologia das plantas. In: SEMINÁRIO SOBRE CORRETIVOS DA ACIDEZ DO SOLO, 2., 1989, Santa Maria. Anais... Santa Maria : UFSM, 1989. p. 39-61.

KIRKPATRICK, H. C.; THOMPSON, J. M.; EDWARDS, J. H. Effects of aluminum concentration on growth and chemical composition of peach seedlings. HortScience, Alexandria, v. 10, p. 132-134, 1975.

KOTZÉ, W. A. G. The effect of aluminium on plant growth and its amelioration in acid subsoils of the Western Cape. Stellenbosch : University of Stellenbosch, 1974. 100 p. Ph.D. Thesis.

KOTZÉ, W. A. G.; DU PREEZ, M. Soil amelioration and fertilization for apple production in South Africa. Acta Horticulturae, Leuven, n. 232, p. 167-176, 1988.

KOTZÉ, W. A. G.; JOUBERT, J. Effect of soil reaction and liming on the growth and mineral nutrition of apple trees. Agrochemophysica, Pretoria, v. 12, p. 15-20, 1980.

MARSCHNER, H. Mineral nutrition of higher plants London : Academic, 1986. 674 p.

MASSOT, N.; POSCHENRIEDER, C.; BARCELÓ, J. Differential response of three bean (Phaseolus vulgaris L.) cultivars to aluminum. Acta Botanica Neerlandica, Stuttgart, v. 41, p. 293-298, 1992.
NASCIMENTO, C. W. A.; SOUZA JÚNIOR, J. O.; MARTINEZ, H. E. P.; TEIXEIRA, P. R. G.; FONTES, P. C. R. Absorção de $\mathrm{P}$ e Al em cafeeiro (Coffea arabica) cultivado em solução nutritiva com sistema radicular parcialmente submetido à doses de alumínio. Revista Ceres, Viçosa, v. 44, n. 253, p. 331-338, 1997.

PASSO, R. R.; RUIZ, H. A. Tolerância dos cafeeiros Conilon e Catuí à toxidez causada pelo alumínio e manganês. Revista Ceres, Viçosa, v. 42, p. 45-52, 1995.

PAVAN, M. A.; BINGHAM, F. T. Toxicity of aluminium to coffee seedlings grown in nutrient solution. Soil Science Society of America Journal, Madison, v. 46, p. 993 997, 1982

PEGTEL, D. M. Responses of plants to $\mathrm{Al}, \mathrm{Mn}$ and Fe, with particular reference to Succisa pratenses Moench. Plant and Soil, Dordrecht, v. 43, p. 43-55, 1986

PEREIRA, J. S.; DANTAS, A. C. M.; ROSSI, A.; FORTES, G. R. L.; VAHL, L. C. Efeito de diferentes concentrações de alumínio na multiplicação e enraizamento in vitro da macieira, cultivar "Marubakaido" (Malus prunifolia Willd, Borkh.). Agropecuária de Clima Temperado, Pelotas, v. 1, n. 1, p. 21-27, 1998.

RENGEL, Z. Role of calcium in aluminium toxicity. New Phytologist, Cambridge, Grã-Bretanha, v. 121, p. 499-513, 1992.

SIVAGURU, M.; JAMES, M. R.; AMBUDURAL, P. R.; BALAKUMAR, T. Characterization of differential aluminum tolerance among rice genotypes cultivated in South India. Journal of Plant Nutrition, New York, v. 15, p. 233-246, 1992

SIVAGURU, M.; PALIWAL, K. Differential aluminum tolerance in some tropical rice cultivars. I. Growth performance. Journal of Plant Nutrition, New York, v. 16, p. $1705-1716,1993$.

SMITH, C. A.; BAILEY, C. H.; HOUGH, L. F. Methods for germinating seeds of some fruit species with special reference to growing seedlings from immature embryos. Piscataway : Rutgers University, 1969. 22 p. (Bulletin, 823).

SUZUKI, A. Doses e modos de aplicação de calcário em pomares de macieira em Latossolo Húmico distrófico do Estado de SC. Viçosa : UFV, 1989. 75 p. Dissertação de Mestrado. 